\title{
Complex-Valued Independent Component Analysis of Natural Images
}

\author{
Valero Laparra ${ }^{1}$, Michael U. Gutmann ${ }^{2}$, Jesús Malo ${ }^{1}$, and Aapo Hyvärinen ${ }^{2}$ \\ 1 Image Processing Laboratory (IPL), \\ Universitat de València, Spain \\ 2 Department of Computer Science \\ Department of Mathematics and Statistics \\ Helsinki Institute for Information Technology HIIT \\ P.O. Box 68, FIN-00014 University of Helsinki, Finland
}

\begin{abstract}
Linear independent component analysis (ICA) learns simple cell receptive fields from natural images. Here, we show that linear complexvalued ICA learns complex cell properties from Fourier-transformed natural images, i.e. two Gabor-like filters with quadrature-phase relationship. Conventional methods for complex-valued ICA assume that the phases of the output signals have uniform distribution. We show here that for natural images the phase distributions are, however, often far from uniform. We thus relax the uniformity assumption and model also the phase of the sources in complex-valued ICA. Compared to the original complex ICA model, the new model provides a better fit to the data, and leads to Gabor filters of qualitatively different shape.
\end{abstract}

Keywords: Complex Independent Components Analysis, Natural Image Statistics, Modeling Fourier phase distribution.

\section{Introduction}

Natural image statistics has become a very useful tool in order to understand how the visual part of the brain works (see for instance 1 for review). One of the most relevant revelations has been that a set of linear sensors optimized to obtain independent sources from natural image data resembles the Gabor-like receptive fields in the primary visual cortex (V1) [2]3].

In recent years, the advances in natural image statistics have been mainly in describing the statistics of the signals after this linear "simple cell" stage 4567789. A common point of these models is that they focus on the total magnitude of the sensor (simple cell) outputs. Often, a combination of the squared outputs of simple cells is learned, leading to something like complex cells. However, there is evidence that relative magnitude, or phase, of simple cells plays an important role. A simple example about the relative importance of the magnitude and phase can be found in 10 . In this example the magnitude and the phase in the Fourier domain of two images were exchanged, and the images which were perceptually more similar to the originals were the ones that carried the phase information. Moreover there is experimental evidence of phase coupled Gabor-like filters in V1 [1211. For this reason, Daugman [13] suggested 
that the receptive field in the first stage could be seen as Gabor sensors defined in the complex domain: the real and the imaginary part are essentially the same Gabor filter but with phases in quadrature.

Despite the evidence of the importance of the phase, not too much progress has been made in statistically modeling the phase of the signals after the simple cell step. The contributions in this field are restricted to models with a fixed linear stage, the wavelet transform 1415. Although this led to interesting results about the distribution of natural images, the statistics used in this kind of modeling could depend on the particular choice of using the wavelet transform as first linear stage.

Here, we aim at both modeling the phase distribution and learning the first linear stage from the data. For that purpose, we are proposing an extension of complex independent component analysis (cICA) [16]. The proposed extension deals with explicitly modeling the phase of non-circularly symmetric sources as an alternative to [17, which does consider non-symmetric sources but it does not model the lack of symmetry.

The paper falls naturally in two parts. In Section 2 , we review cICA and point out its limitations in modeling the phase distribution. Section 3 shows how cICA can be extended to better capture the distribution of the phase variable. The extension includes the version of [16] as special case. Although we focus here on natural images, the extension can be applied to all kinds of data. Conclusions are drawn in Section 4.

\section{Complex Independent Component Analysis and Its Limitations}

As in Independent Component Analysis (ICA) for real variables, the goal in complex ICA (cICA) [16] is to find a linear transformation $W$ such that, when applied to some vector of signals $x$, the elements of the output vector $s=W^{H} x$ are statistically as independent as possible. The difference to real ICA is that $W$, $x$, and hence also $s$ are complex valued. Furthermore, instead of the transpose $W^{T}$, the transposed, complex conjugate $W^{H}$ is used.

In ICA, one approach to find such a $W$ is to first whiten the data and then to maximize the kurtosis, or a statistically more robust contrast function. In cICA, the same approach can be taken by appropriately defining whitening and choosing an appropriate contrast function.

For complex variables, the random vector $x$ is white if both the real and imaginary part are white and if the real and imaginary parts are uncorrelated. An equivalent condition is that $\mathbb{E}\left\{x x^{H}\right\}=I$ and $\mathbb{E}\left\{x x^{T}\right\}=0$. Denoting a column of $W$ by $w_{i}$, in [16], cICA can be performed by optimization of $J_{G}$,

$$
J_{G}(W)=\sum_{i=1}^{n} \mathbb{E}\left\{G\left(\left|w_{i}^{H} x\right|^{2}\right)\right\},
$$


under the constraint $W^{H} W=I$. Depending on the nature of the sources, $J_{G}$ needs to be maximized or minimized. The contrast function $G$ must be a smooth even function and $x$ is assumed to be white. Possible candidates include $G(y)=-\sqrt{a+y^{2}}$ for a small constant $a$. In the simulations in the next section, we will use this contrast function with $a=0.1$. Note that the objective function depends only on the moduli $r_{i}=\left|w_{i}^{H} x\right|$ of the complex variable $s_{i}=w_{i}^{H} x$, no matter the choice of $G$. For sparse sources, maximization of this $G$ leads to consistent estimators [16].

An alternative viewpoint of cICA is based on maximum likelihood estimation of the statistical model $s=W^{H} x$ where $x$ and $s$ are white and $W^{H} W=I$. Assuming independence of the sources in $s=\left(s_{1}, \ldots, s_{n}\right)^{T}$, the log-likelihood is

$$
\ell(W)=\sum_{t} \sum_{i=1}^{n} \log p_{s_{i}}\left(w_{i}^{T} x_{t}\right)
$$

where $x_{t}$ is the $t$-th observation of $x$ and $p_{s_{i}}$ is the density of the sources $s_{i}$. Since the variables are complex valued, $p_{s_{i}}\left(s_{i}\right)$ is a bidimensional distribution that can be written as $p_{r \phi}\left(r_{i}, \phi_{i}\right) / r_{i}$, where $r_{i}$ is the modulus and $\phi_{i}$ is the phase of $s_{i}$. Assuming further that the modulus and the phase are independent and that, importantly for the next sections, the distribution of the phase is a uniform distribution, maximization of $\ell$ becomes maximization of

$$
J_{2}(W)=\sum_{t} \sum_{i=1}^{n}\left(\log p_{r}\left(r_{i t}\right)-\log r_{i t}\right) .
$$

The term $p_{r}$ denotes the distribution for the moduli $r_{i}$, where we assume that all of them follow the same distribution. Replacing sample average by expectation, we obtain the objective function in Eq1 with $G\left(r^{2}\right)=\log p_{r}(r)-\log r$. Note further that the distribution $p_{q}$ of the squared modulus $q=r^{2}$ is $p_{q}(q)=p_{r}(r) /(2 r)$. This means that $G(q)=\log p_{q}(q)+\log 2$. Hence, the contrast function $G$ used in cICA can be directly related to the distribution of the squared moduli of the complex sources. In particular, we can relate the contrast function $G(q)=-\sqrt{a+q^{2}}$, where $a$ is a small constant, to the choice of $p_{q}$ being a Gamma distribution,

$$
p_{q}(q)=q^{k-1} \frac{\exp \frac{-q}{\theta}}{\Gamma(k) \theta^{k}}
$$

with $k=1$. Then, $\log p_{q}(q)=-q+$ const, which is, up to additive constants, the same as the above contrast function when $a$ is small.

\subsection{Simulations with Natural Images}

We apply cICA on natural images in the Fourier domain. The natural images are $16 \times 16$ patches extracted from the data base in [18. The data $x$ on which we apply cICA are the complex Fourier coefficients. For the visualization, we show the obtained filters, which correspond to: the learned matrix $W$, combined with the whitening matrix, and the Fourier transform. 

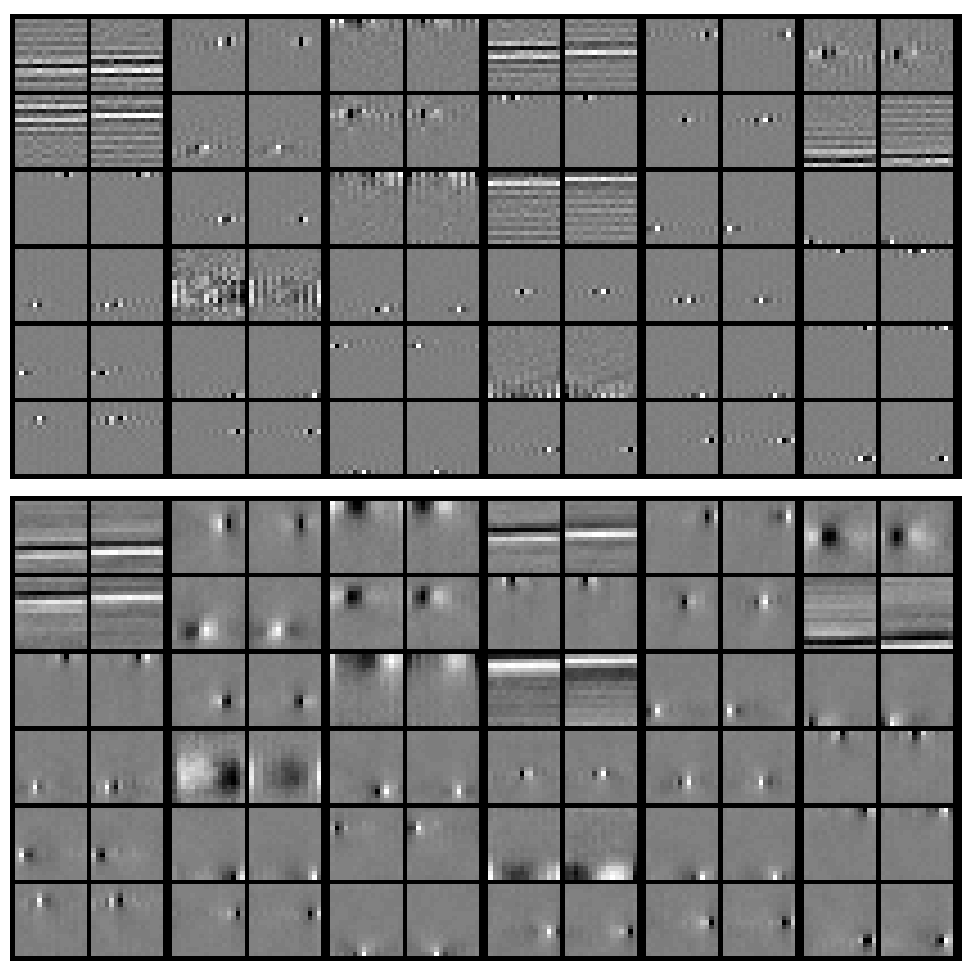

Fig. 1. Filters (see text for details) and features (defined by the pseudoinverse of the filter matrix) obtained with cICA using the algorithm in [16], ordered according to their contribution to the value of $J_{G}$ in Eq. 1 (first 36 of 126). Filters and features are shown in pairs, with the real part at the left and the imaginary part at the right. Top: complex filters. Bottom: complex features.

Fig. 1 shows the results. The real and the imaginary part of the learned complex filters are shown in pairs from left to the right. Real and imaginary part are Gabor-like filters that are in quadrature-phase. This statistical result is in line with measurements in V1 1211] and related empirical models [13.

Complex ICA results essentially replicate those obtained by independent subspace analysis [4. It is the complex-valued formalism which allows to automatically create two-dimensional subspaces with a linear model formulation as in ordinary ICA.

\subsection{Checking Model Assumptions}

Here we check whether, for natural images, the obtained complex sources $s_{i}$ follow the assumption in cICA that the (squared) moduli follow a Gamma distribution and the phases are uniformly distributed.

Fitting gamma distributions to the empirical distributions of the modulus of the sources leads to good fits, see Fig. 2, In contrast, the empirical distributions 
of the phases do not follow the model assumptions, as shown in Fig. 3. The clearly visible oscillations in the phases violate the assumption of uniformity in cICA. These roughly bimodal histograms may be modeled by a modified von Mises distribution to account for the two peaks,

$$
p_{\phi}(\phi \mid k, \mu)=\frac{1}{2 \pi I_{0}(k)} e^{k \cos (2(\phi-\mu))},
$$

where $I_{0}(k)$ is the Bessel function of order 0. In contrast to the ordinary von Mises distribution, we have here introduced the factor 2 inside the cosine to model the two-peaked distributions seen in Fig. 3. Note that this distribution correspond to a uniform distribution when the parameter $k=0$. In Fig. 3] we can see how fitting this distribution to the empirical distribution of the phase is much more precise than fitting a uniform distribution.

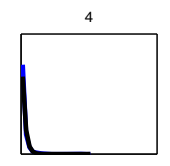

17

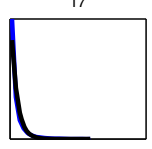

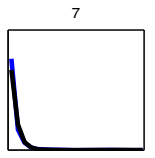

20

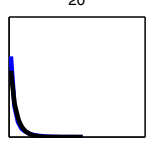

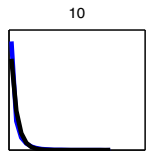

22

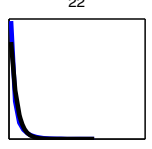

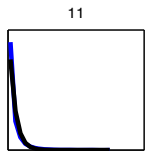

23

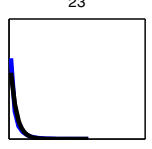

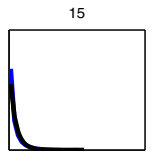

28

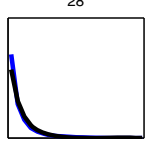

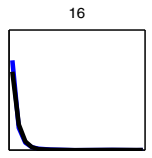

31

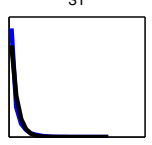

Fig. 2. Selection of distributions of the modulus of the cICA sources (blue) and the fitted gamma distributions (black). The curves are strongly overlapping. Numbers refer to the corresponding filters in Fig. 1 (left to right, top to bottom).

\section{Extension of Complex ICA}

In this section we propose an extension of cICA. The extension builds on the maximum likelihood approach to cICA in Eq. 2. It will take into account that the distribution of the phase variables can be non-uniform, as found in natural images (Eq. [5] and Fig. [3).

As in the previous section, we write in Eq. 2, $p_{s_{i}}$ as $p_{r \phi}\left(r_{i}, \phi_{i}\right) / r_{i}$, where $r_{i}$ is the modulus and $\phi_{i}$ is the phase of $s_{i}$. Also as previously, we assume that the modulus and the phase are independent. However, instead of assuming a uniform distribution for the phases, we assume the distribution in Eq. 5. Here, we can set $\mu=0$ because this phase localization parameter is redundant: the phase of the oscillations will be determined by the estimated features anyway. Since this distribution includes the uniform distribution, our extension includes the conventional cICA as a special case. With these assumptions, the maximum likelihood principle leads us to maximize the following objective function

$$
J_{G Q}\left(W, k_{i}\right)=\sum_{i} \mathbb{E}\left\{G\left(r_{i}\right)+Q\left(\phi_{i}, k_{i}\right)\right\} .
$$




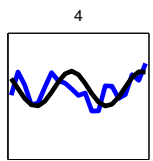

17

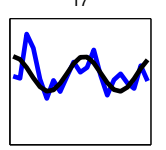

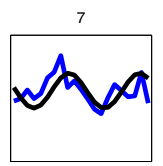

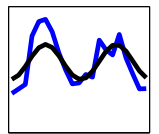

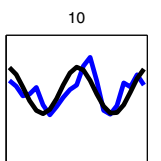

22

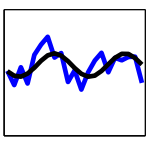

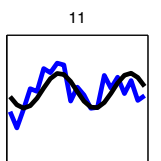

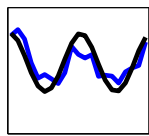

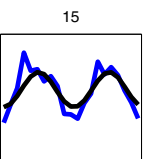

28

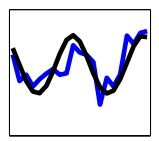

16
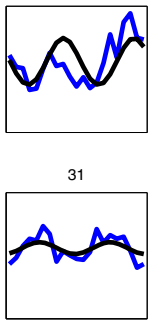

Fig. 3. Selection of distributions of the phases of the cICA sources (blue) and the fitted modified von Mises distributions (black). Numbers refer to the corresponding filters in Fig. 1 (left to right, top to bottom).
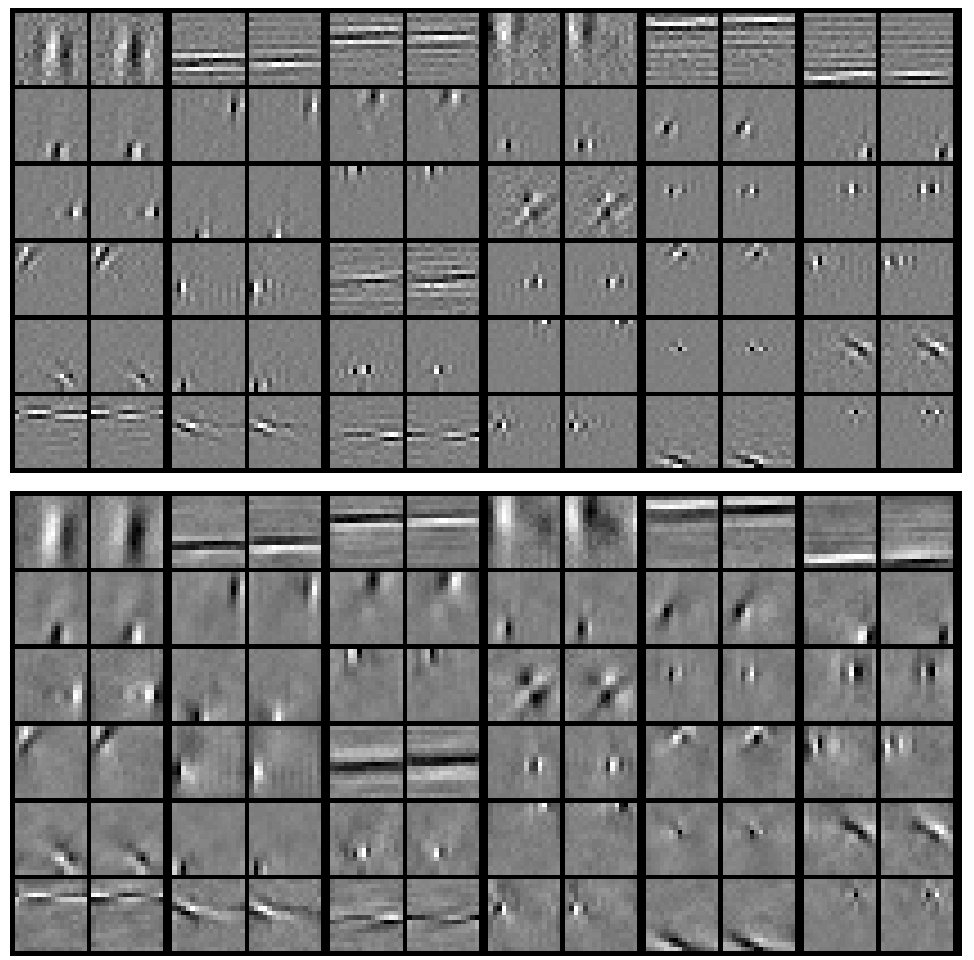

Fig. 4. Filters and features obtained with the extended cICA, ordered according to ctheir contribution to the value of $J_{G Q}$ in Eq. 6 (first 36 of 126). Filters and features are shown in pairs with the real part on the left and the imaginary part on the right. Top: complex filters. Bottom: complex features.

Here, $r_{i}$ is the modulus of the complex number $w_{i}^{H} x$, and $\phi_{i}$ is its phase. As before, $w_{i}$ denotes a column of the matrix $W$ and we have the constraint $W^{H} W=I$. The function $G$ is, as before, related to the distribution of the 


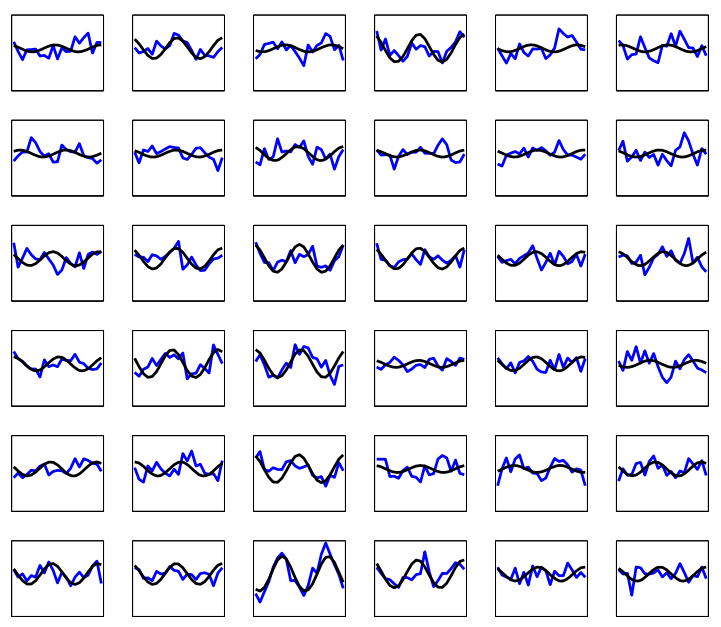

Fig. 5. Phase distributions of the sources obtained using the modified cICA algorithm. The distribution of the phases corresponding to the filters in Fig. 4 are shown. Empirical distributions are plotted in blue and fitted modified Von Mises distribution in black.

squared modulus. A possible choice is $G(y)=-\sqrt{a+y^{2}}$. The function $Q$ is related to the distribution of the phase and is given by $Q\left(\phi_{i}, k_{i}\right)=k_{i} \cos \left(2 \phi_{i}\right)$, and depends on the $w_{i}$ and the shape parameters $k_{i}$.

This modification of cICA can also be considered from an information theoretical point of view. The main goal of all ICA-based algorithms is to obtain independent sources, which is equivalent to reduce the mutual information (MI) between them. Therefore, as $M I\left(s_{1}, s_{2}, \ldots, s_{n}\right)=\sum_{i}\left\{h\left(r_{i}\right)+h\left(\phi_{i}\right)\right\}-h\left(s_{1}, s_{2}, \ldots, s_{n}\right)$, where $h(\cdot)$ is the entropy. This result can be derived by using the same assumptions as in section 2 Accordingly, we have to reduce the entropy of $r_{i}$ and $\phi_{i}$, (the joint entropy is invariant under unitary transforms). Note that the uniform distribution is the one with maximum entropy when the domain is bounded. Therefore, anything different to a uniform phase distribution will have less entropy, which means less MI between the variables, and hence more independent sources.

Fig. 4 shows the results when the above extended cICA is applied to natural images (same setup as before). Note how the shape of the filters is more elongated (especially the highest-ranked ones) and spatially more extended than for the classical cICA. In Fig. 5 we can see the distribution of phases of the sources obtained with the proposed algorithm. The distributions are similar to the proposed modified von Mises distribution.

\section{Conclusions}

In this paper, we have started with modeling natural images with complex Independent Component Analysis (cICA). This led to the emergence of complex 
filters where the real and the imaginary parts have the same Gabor-like shape (same orientation and same frequency) but a phase difference of $\frac{\pi}{2}$.

Checking the model assumptions in cICA, we have noticed that the assumption of uniformity of the phases is often violated for natural image data. This led us to formulate an extension of cICA which models also the phase distributions. Simulations with natural images showed that the empirical distribution of the phases provide a good match to the assumptions of the extended model.

Our research has the potential for more extensions. For instance, the assumption of the independence between modulus and phase should be investigated more carefully.

\section{References}

1. Simoncelli, E.P., Olshausen, B.A.: Natural image statistics and neural representation. Annual Review of Neuroscience 24(1), 1193-1216 (2001)

2. Olshausen, B., Field, D.: 'Emergence of simple-cell receptive field properties by learning a sparse code for natural images. Nature 381, 607-609 (1996)

3. Bell, A.J., Sejnowski, T.J.: 'The 'Independent Components' of Natural Scenes are Edge Filters. Vision Research 37(23), 3327-3338 (1997)

4. Hyvärinen, A., Hoyer, P.: Emergence of phase- and shift-invariant features by decomposition of natural images into independent feature subspaces. Neural Computation 12(7), 1705-1720 (2000)

5. Portilla, J., Strela, V., Wainwright, M., Simoncelli, E.: Image denoising using scale mixtures of Gaussians in the wavelet domain (2003)

6. Hyvärinen, A., Hurri, J., Hoyer, P.O.: Natural Image Statistics. Springer, Heidelberg (2009)

7. Lyu, S., Simoncelli, E.P.: Nonlinear extraction of independent components of natural images using radial gaussianization. Neural computation 21(6), 1485-1519 (2009)

8. Eichhorn, J., Sinz, F., Bethge, M.: Natural image coding in V1: how much use is orientation selectivity? PLoS computational biology 5(4) (2009)

9. Malo, J., Laparra, V.: Psychophysically Tuned Divisive Normalization factorizes the PDF of Natural Images. Neural Computation 22(12) (2010)

10. Oppenheim, A.V., Lim, J.S.: The importance of phase in signals. Proceedings of the IEEE 69(5), 529-541 (1981)

11. Touryan, J., Felsen, G., Dan, Y.: Spatial structure of complex cell receptive fields measured with natural images. Neuron 45(5), 781-791 (2005)

12. Pollen, D.A., Ronner, S.F.: Phase relationships between adjacent simple cells in the visual cortex. Science 212(4501), 1409-1411 (1981)

13. Daugman, J.G.: Quadrature-phase simple-cell pairs are appropriately described in complex analytic form. J. Opt. Soc. Am. A 10(2), 375-377 (1993)

14. Portilla, J., Simoncelli, E.P.: A Parametric Texture Model Based on Joint Statistics of Complex Wavelet Coefficients. International Journal of Computer Vision 40(1), 49-70 (2000)

15. Cadieu, C.: Probabilistic Models of Phase Variables for Visual Representation and Neural Dynamics, Ph.D. thesis, UC Berkeley (2009)

16. Bingham, E., Hyvärinen, A.: A fast fixed-point algorithm for independent component analysis of complex valued signals. International Journal of Neural Systems 10, $1-8(2000)$

17. Eriksson, J., Seppola, A.M., Koivunen, V.: Complex ICA for circular and noncircular sources. In: Proc. EUSIPOCO (2005)

18. Olmos, A., Kingdom, F.A.: Mcgill Calibrated Color Image Database (2004) 